\title{
PIV and LIF study of flow and thermal fields of twine plumes in water
}

\author{
Zuzana Broučková ${ }^{1}$ and Zdeněk Trávníček ${ }^{1}$ \\ ${ }^{1}$ Institute of Thermomechanics, The Czech Academy of Sciences, Prague, Czech Republic
}

\begin{abstract}
Flow and thermal fields of a pair of plane plumes in water are investigated by means of PIV and LIF experiments. The plumes are generated from thermal line sources, which are made out of electrically heated cylinders with a diameter of $D=1.21 \mathrm{~mm}$. A cylinder-to-cylinder distance was $17.9 \mathrm{D}$. Either continuous or pulsating heating were used with the same heating input power. Because the cylinder-tocylinder distance is moderately small, deflections of plumes from a vertical direction occur and the plumes are inclined together. This behavior is caused by a confined entrainment from a space between the both plumes. For a continuous heating, low frequency oscillations were identified and the natural frequency was evaluated as $0.5 \mathrm{~Hz}$. Based on this finding, pulsating heating was used at the subharmonic frequency of 0.25 Hz. The maximum time-mean velocity magnitude at the continuous and pulsating heating were commensurable, approximately $0.007 \mathrm{~m} / \mathrm{s}$. On the other hand, pulsating heating achieves by $36 \%$ higher velocity peaks. A very strong velocity oscillations were generated by pulsating heating at the distance approximately $8.3 \mathrm{D}$ above the cylinders, where the velocity maxima oscillate along the time-mean value of $0.0057 \mathrm{~m} / \mathrm{s}$ from $-30 \%$ to $+70 \%$. Temperature fields reasonably agree with this findings, despite a relatively fast equalization of the temperature field was concluded. The results demonstrate enhancement effects of pulsations in flow/thermal fields.
\end{abstract}

\section{Introduction}

A buoyant jet is a type of fluid motion and one of basic examples of free shear flows - see Schlichting [1], Blevins [2]. It is generated by differences in fluid density. In other words, the driven force (buoyancy) results from density differences. A buoyant jet whose initial momentum is approximately zero is called a plume. In the case of a thermal source, the plume is developed because a local temperature increase causes a decrease of density. For a line thermal source, a plane plume is developed. In the present study, a twin of plane plumes has been investigated by means of PIV and LIF experiments in water.

\section{Experiments}

\subsection{Experimental setup and methods}

Figure 1 shows a schematic view of the present experimental setup. The experiments are made in a glass water tank with the following dimensions: height $\times$ length $\times$ depth $=150 \times 245 \times 140 \mathrm{~mm}$. The working fluid is distilled water.

The device frame is made out of PMMA (polymethyl methacrylate). A pair of line thermal sources (pos. 1 in Fig. 1) are used to generate a twine of the plane plumes. These thermal sources are made from a smooth stainless steel tubes, its outside and inside diameters are $D=1.21 \mathrm{~mm}$ and $0.91 \mathrm{~mm}$, respectively. The cylinders are mounted horizontally at the center-tocenter distance of $21.7 \mathrm{~mm}$. Four metal brackets (2) fix the cylinders at the ends. The total length of the cylinders between the brackets is $L=60.7 \mathrm{~mm}$, i.e. the aspect ratio is $L / D=50.2$. The immersion depth of cylinders is approximately $40 \mathrm{~mm}$. The coordinate system $x, y, z$ is also shown in Fig. 1 .

The cylinders are heated by the Joule effect of direct or pulsating current $I$ from the brackets and Fig. 1 schematically shows the electrical connections. The PCcontrolled power supply (Voltcraft CPPS, maximum DC parameters: $42 \mathrm{~V}, 20 \mathrm{~A}$, and $320 \mathrm{~W}$ ) can operate in two regimes to produce either direct or pulsating current. The maximum current was $12.9 \mathrm{~A}$ in this study.

For DC-supply heating (continuous heating, $\mathrm{CH}$ ), the current $I$ is measured by a multimeter (Metex $\mathrm{M}$ $4600 \mathrm{M})$. Voltage differences along the cylinders $\left(V_{\mathrm{L}}\right.$ and $V_{\mathrm{R}}$ ) are measured by two multimeters (Metex M 3800) via the 4-terminal sensing (considering that the 4-point probes method allows more accurate measurements). The total voltage difference is $V=V_{\mathrm{L}}+V_{\mathrm{R}}$. The maximum uncertainties of the current and voltage measurements were less than $\pm 0.3 \%$, and $\pm 0.5 \%$, respectively.

For pulsating-supply heating (pulsating heating, $\mathrm{PH}$ ), the current and voltage are measured by means of

Corresponding author: brouckova@it.cas.cz

C The Authors, published by EDP Sciences. This is an open access article distributed under the terms of the Creative Commons Attribution License 4.0 (http://creativecommons.org/licenses/by/4.0/). 

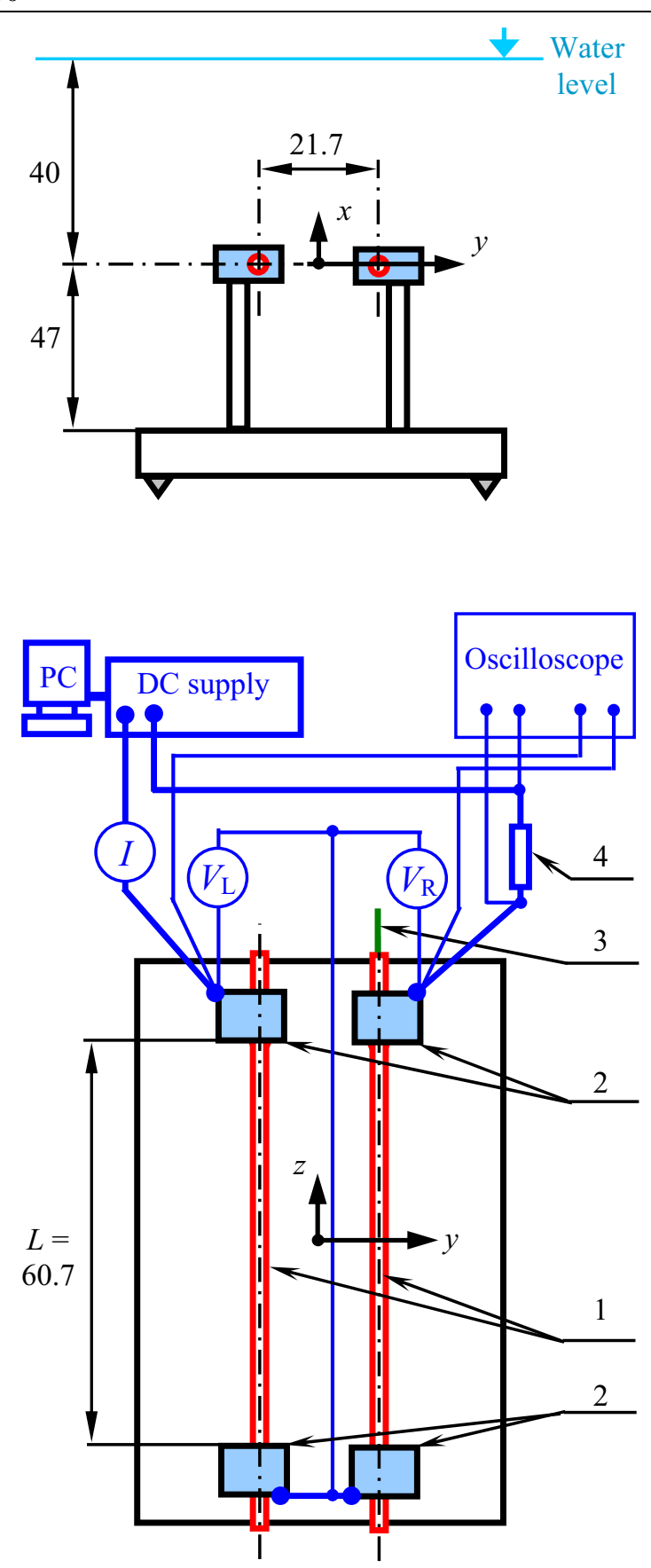

Fig. 1. Experimental device, sizes are in millimetres; 1: heated cylinders, 2: brackets, 3: thermocouples for $T_{\mathrm{w}}$ measurement, 4: shunt $0.100 \Omega$.

the two channel oscilloscope (Agilent DSO3102A) with a $0.100 \Omega$ shunt (4) - see Fig. 1 .

The LIF technique is based on the fluorescence effect. A solution of Rhodamine $B$ is inserted into the water. The dye-stained flow is lit by the laser sheet (Nd:Yag pulsed laser, Litron, NANO S 65-15, with a wavelength of $532 \mathrm{~nm}$ and $65 \mathrm{~mJ}$ output energy of a 6$8 \mathrm{~ns}$ pulse, with cylindrical optics). The laser is placed approximately $1.8 \mathrm{~m}$ from the water tank. The repetition rate is $15 \mathrm{~Hz}$ (i.e. the maximum of the laser possibility). The flow field pictures are recorded with a digital camera (HiSense Neo, 2560×2160 pixels, 16 bit) equipped with the lens (Tokina $100 \mathrm{~mm}$ F2.8 Macro D) and an optical filter (high pass in wavelength with cut-

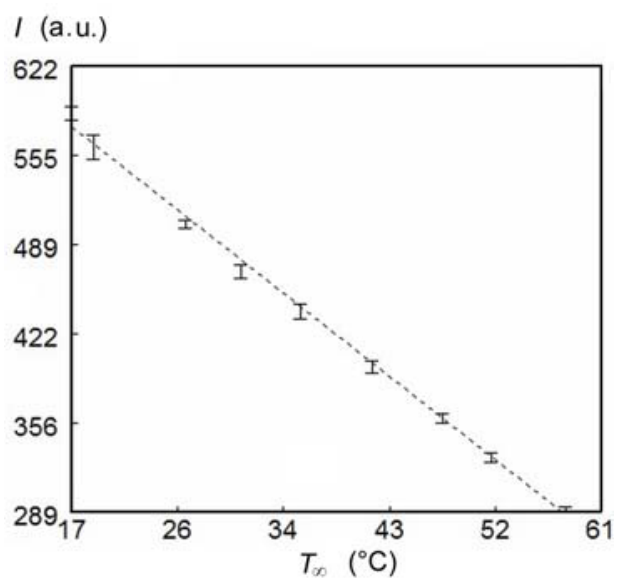

Fig. 2. The relation between the fluorescence intensity and the temperature.

off $570 \mathrm{~nm}$ ) which allows transmission of the fluorescent light only while filters out the laser-scattered light. The camera is placed approximately $350 \mathrm{~mm}$ from the water tank, producing approximately $62 \times 52 \mathrm{~mm}$ field of view. The system is controlled by DynamicStudio v3.40 Software (Dantec Dynamics). More details were described previously by Broučková et al. [3].

To quantify temperature field by means of the LIF technique, an in-situ calibration was performed in the range of $17-58^{\circ} \mathrm{C}$. A typical calibration curve is shown in Fig. 2 as a relationship between the fluorescence intensity (in arbitrary units) and temperature at the given laser conditions and given Rhodamine B concentration. To enhance reliability of the calibration experiment, each calibration point were measured repeatedly three times and the error bars in Fig. 2 indicate reasonably small data scattering (causing mainly by a slight variation of the laser intensity with time). The relationship is linear in character which well agrees with expectations based on literature results; see e.g. Seuntiëns et al. [4].

The PIV experiments are made with the same techniques (laser, camera with the color filter, and software) as described in the text above. Fluorescent seeding particles (PS-FluoRed-FRAK-Fi204, 1-20 $\mu \mathrm{m}$ in diameter) are used. The wavelength peaks of the absorption and emission lights are $530 \mathrm{~nm}$ and $607 \mathrm{~nm}$, respectively. The adaptive PIV technique is used with the minimum and maximum interrogation areas $32 \times 32$ pixels and $64 \times 64$ pixels, respectively. The resulting velocity maps are averaged from a sequence of 50 or 90 images.

The temperature of the right cylinder $\left(T_{\mathrm{W}}\right)$ is measured by a J type (iron-constantan) thermocouple connected with a thermometer Omega DP41-B. The thermocouple was inserted through the cylinder tube on the coordinate $z=0$, see pos. 3 in Fig. 1 . The temperature difference between inside and outside of cylinder surface has been neglected since the cylinder wall had only $0.15 \mathrm{~mm}$ thickness. Therefore, the measured temperature $T_{\mathrm{w}}$ is considered as the average surface temperature of the cylinder. A similar approach was used in the recent forced convection study by Broučková and Trávníček [5].

The bulk temperature of quiescent water in the tank $T_{\infty}$ is evaluated as the average of two temperatures, 
measured by two NTC thermistor sensors connecting width a thermometer (Ahlborn AMR, Therm 2280-3). The sensors are placed below the tested cylinders, approximately in the places of $x=-30 \mathrm{~mm}, \mathrm{y}= \pm 11 \mathrm{~mm}$ and $z=-10 \mathrm{~mm}$. During the present experiments, the maximum differences between the both bulk temperatures were $0.3^{\circ} \mathrm{C}$. The temperatures of the cylinders and water ranged $T_{\mathrm{w}}=31-64^{\circ} \mathrm{C}$ and $T_{\infty}=23-$ $26{ }^{\circ} \mathrm{C}$, respectively. All the thermometers with probes were calibrated and the maximum uncertainties of the $T_{\mathrm{w}}$ and $T_{\infty}$ are less than $\pm 0.2^{\circ} \mathrm{C}$ and $\pm 0.1^{\circ} \mathrm{C}$, respectively.

\subsection{Parameters and data reduction}

The heating input power $P$ is evaluated from the measured electric current $I$ and voltage $V$. For DCsupply heating (continuous heating, $\mathrm{CH}$ ), the input power is $P=I \cdot V$. For pulsating-supply heating (PH), the input power can be evaluated by integration

$$
P=\int_{0}^{T} i(t) v(t) \mathrm{d} t
$$

where $i(t)$ and $v(t)$ are the instantaneous current and voltage, $t$ is time and $T$ is the cycle period. In the present case, the rectangular signal is used with the electric current and voltage alternating between and the values $I$ an $V$, respectively, and zeros. The duty cycle was chosen $50 \%$, thus the heating is off/on during the cycle parts of $t / T=0-0.5 / 0.5-1.0$, respectively. Therefore the input power can be evaluated as $P=I \cdot V / 2$. The experiments were made in a range of $P=6-41 \mathrm{~W}$, and the present study deals with the case of $P=14.7 \mathrm{~W}$.

Based on an experimental evaluation of the natural frequency of the present plumes, the thermal actuation was chosen as a subharmonic action with the driven frequency of $f=1 / T=0.25 \mathrm{~Hz}$; more details of this approach and findings are explained in part 3.1 bellow.

The measured temperature $T_{\mathrm{w}}$ is the average surface temperature of the cylinder, as written in the text above. The thermal boundary condition at the cylinder surface is approximately uniform heat flux, similarly as in [5]. Small differences from the heat flux uniformity which result from the local temperature differences are negligible because of small sensitivity of the stainless steel electrical resistivity to temperature. Namely, the temperature coefficient of the resistivity of the present stainless steel was evaluated as $0.0009{ }^{\circ} \mathrm{C}^{-1}$, hence the temperature differences of order $10^{\circ} \mathrm{C}$ can cause the resistivity changes less than $1 \%$. Thus the Joule's first law gives negligible differences (less than $1 \%$ ) in the local heat generation rates and local heat fluxes.

The governing parameter for natural convection is the Grashof number, which is defined as $G r=g \beta\left(T_{\mathrm{w}}-T_{\infty}\right) D^{3} / v^{2}$, where $g$ is the acceleration of gravity and $\beta$ is the volumetric thermal expansion coefficient. The both material parameters $\beta$ and $v$ are evaluated at the film temperature $T_{\mathrm{f}}$.

Another parameter important in natural convection is the Rayleigh number, which is defined as $R a=G r P r$, where $\operatorname{Pr}$ is the Prandtl number. Again, an evaluation of the $P r$ is made at the film temperature $T_{\mathrm{f}}$.

\section{Results and discussion}

The following experiments were performed after well stabilized flow and temperature fields, i.e. at least 1 hour after filling the water tank with water of the laboratory temperature, and at least 5 minutes after heating was turned on.

\subsection{Velocity flow field: PIV experiment}

The measurements were performed for the investigated twin plumes, which are generated by either continuous or pulsating heating ( $\mathrm{CH}$ or $\mathrm{PH}$, respectively). The operating parameters are summarized in Table 1 including the temperature differences $T_{\mathrm{w}}-T_{\infty}$ and the Grashof and Rayleigh numbers. Note that the both cases of heating, which are presented in this study, have identical heating input power of $P=17.4 \mathrm{~W}$.

Table 1. Operating parameters of the present experiments

\begin{tabular}{lllll}
\hline Case & $f$ & $T_{\mathrm{w}}-T_{\infty}$ & $G r$ & $R a$ \\
& $(\mathrm{~Hz})$ & $\left({ }^{\circ} \mathrm{C}\right)$ & & \\
\hline \hline $\begin{array}{l}\text { CH } \\
\text { (continuous }\end{array}$ & 0 & 19.8 & 203 & 958 \\
heating) & & & & \\
\hline $\begin{array}{l}\text { PH } \\
\text { (pulsating } \\
\text { heating) }\end{array}$ & 0.25 & $17.7-$ & $203-$ & $915-$ \\
\hline
\end{tabular}

Figure 3 shows a typical PIV result of the twin plumes at $\mathrm{CH}$ case as the time-mean velocity vector map colored by the velocity magnitude. Figure 3 was made by averaging over 50 instantaneous flow fields. Two small white crosses indicate the positions of the cylinders (they are not visible here because they are hidden behind their brackets, as can be clear from Fig. 1). A small white ring in Fig. 3 indicates the position in the shear layer in which the velocity magnitude data were analyzed to obtain the time behavior and power spectral density (PSD), as will be discussed in the following text (Figs. 5 and 6).

As is presented in Fig. 3, the plumes issuing from the cylinders are laminar in character. They are inclined together. The main reason is a fluid entrainment from surroundings to the plumes. The entrainments generate a very weak under pressure between the plumes, however the plumes restrict each other to entrain fluid from there. Note that another mechanism causing a deformation of plumes is linked with the 3-dimensional end effects. The fluid entrainments are not confined at the plume ends, therefore the plume volume flux is enhanced at the ends. 

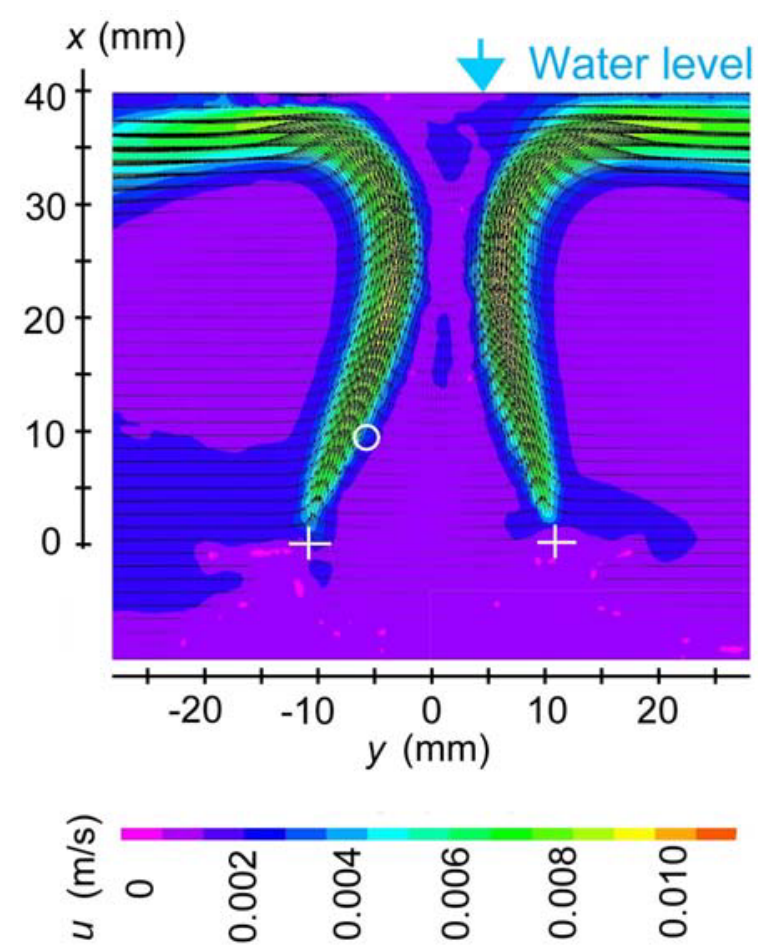

Fig. 3. Time-mean velocities of $\mathrm{CH}$ case, presented as the vector map colored by the velocity magnitude; parameters are summarized in Table 1.

It is worthy of notice here that this mechanism is well know from rectangular fluid jets, where this process basically changes a two-dimensional (plane) jet flow causing a three-dimensional flow character with a saddle-back velocity profile - see Vouros et al. [6] and Broučková et al. [3].

It can be imagined that the both plumes could merge in the far field. However, the present geometry and given moderate immersion depth of cylinders (40 mm i.e. $33 D$, see Fig. 1) causes impingements of the plumes to the water surface and plume turning apart from each other. Finally, a pair of the wall jets is generated to the opposite directions along the water surface.

Figures $4(a-c)$ show the twin plumes generated by pulsating heating $(\mathrm{PH})$. Meaning of the small white crosses and the small white ring are the same as in Fig. 3 , i.e. the cylinders positions and a point for time and frequency analyzes of velocities, respectively. A comparison of the both time-mean cases $\mathrm{CH}$ and $\mathrm{PH}$ (Figs. 3 and 4(a), respectively) indicate more stronger character of the time-mean $\mathrm{PH}$ plumes including wider wall jets outgoing from the test section. To demonstrate more deeply this behavior, Figs. 4(b and c) show the flow fields at heating and non-heating parts of the pulsating-supply cycle, respectively: the time-averaging was made for 10 instantaneous flow fields at $t / T=0.32-$ 0.47 and $0.72-0.87$, respectively. As is shown, ascending flows from the cylinders are generated during the heating moment - see Fig.4(b). Obviously, warmed fluid volumes continue the advection motion during the nonheating moment - see Fig.4(c). The maximum timemean velocity magnitude of $\mathrm{CH}$ and $\mathrm{PH}$ cases are commensurable - they are approximately $0.007 \mathrm{~m} / \mathrm{s}$, see

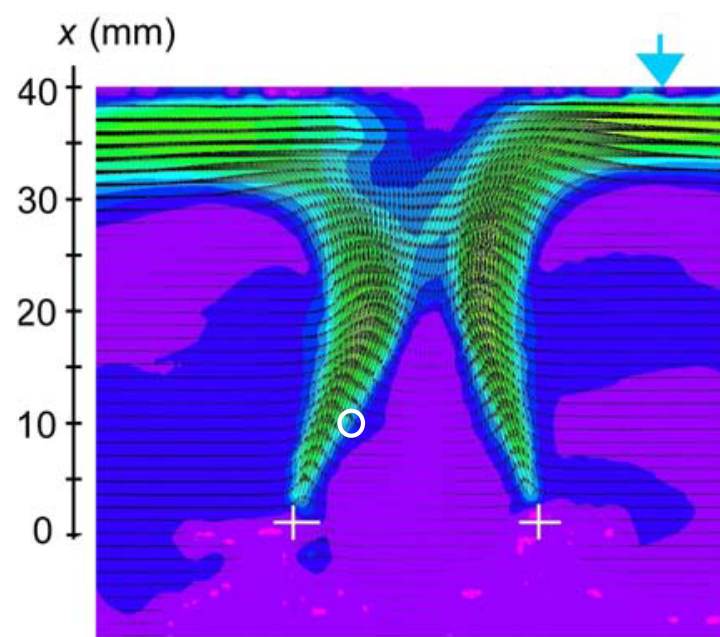

(a)

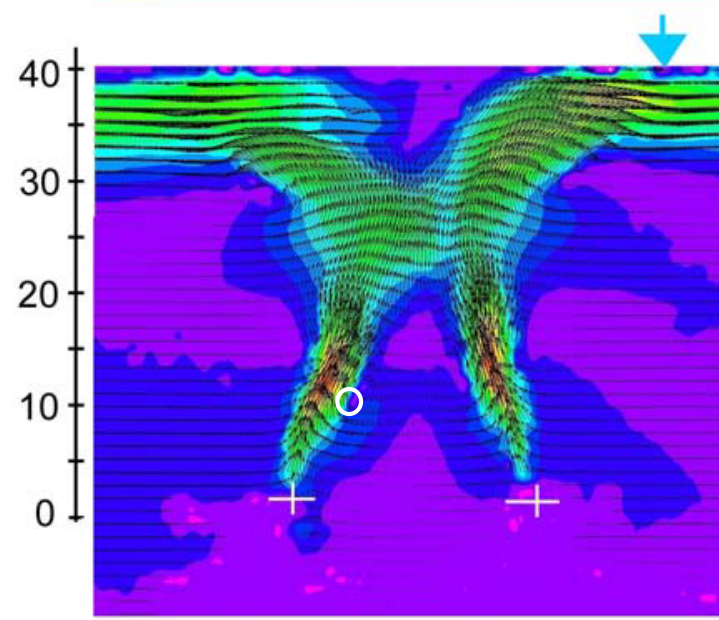

(b)
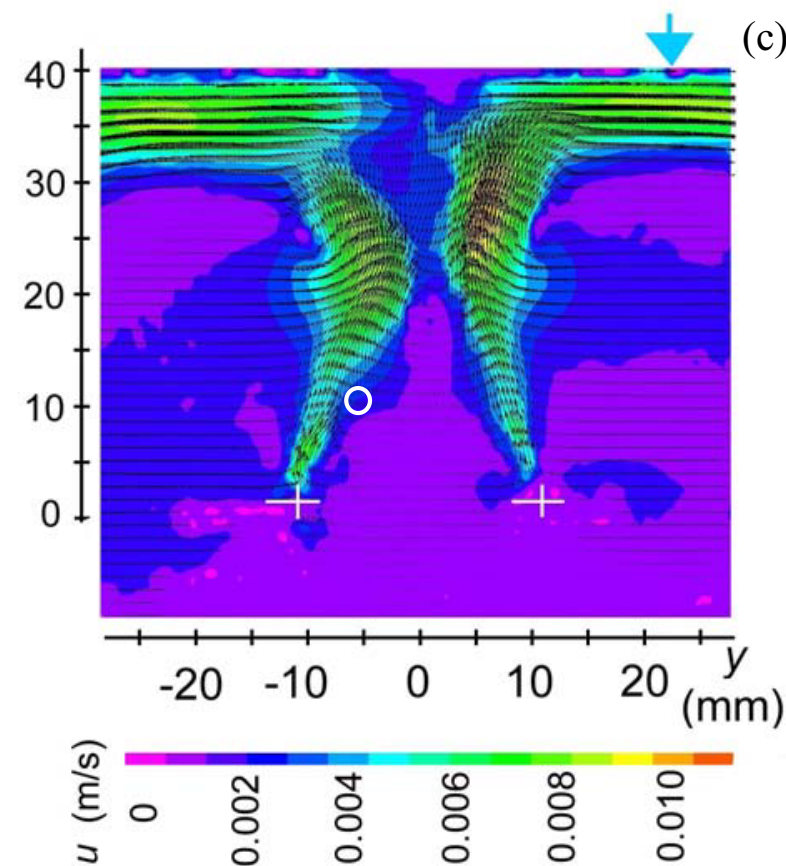

Fig. 4. Velocities of PH case: vector maps colored by the velocity magnitude; (a) time-mean results, (b) time-averaging for heating part of the cycle, at $t / T=0.72-0.87$, (b) timeaveraging for non-heating part of the cycle at $t / T=0.32-0.47$; (parameters - see Table 1).

Figs. 3 and 4(a). On the other hand, the both heating and non-heating moments always generate higher velocities, 
namely 0.010 and $0.008 \mathrm{~m} / \mathrm{s}$, see Figs. 4(b and c), respectively. In other words, the $\mathrm{PH}$ generates by $36 \%$ higher velocity magnitude peaks in comparison with $\mathrm{CH}$ case - see a comparison of Figs. 4(a) and Fig. 3, respectively. An obvious reason is a concentration of the heating input power into one half of the cycle.

Unsteady components of velocities result from the natural frequency of plume flows and from the thermal periodical actuation. The former source can be an exclusive reason of oscillations (if any exists) for $\mathrm{CH}$ case and the latter one can be a prevailing source of oscillations for $\mathrm{PH}$ case. To demonstrate this opinion, a temporal evolution of the velocity magnitude was extracted from the PIV data in a given representative point, located in the shear layer of plumes, where an intense receptivity to disturbances can be expected. Namely, the point was chosen at the coordinates of $x=$ $9.4 \mathrm{~mm}$ and $y=-5.3 \mathrm{~mm}$. This point is marked by a small white ring in Figs. 3 and $4(a-c)$. The temporal evolutions of the velocity magnitude for the both cases $\mathrm{CH}, \mathrm{PH}$ are shown in Fig. 5. The velocity magnitude at $\mathrm{CH}$ oscillates between 0.0015 and $0.006 \mathrm{~m} / \mathrm{s}$. On the other hand, velocities at PH clearly demonstrates the predetermined actuation cycle oscillating between the non-heating and heating parts of the cycle, i.e. from approximately $0.003 \mathrm{~m} / \mathrm{s}$ at $t / T=0-0.5$ to $0.006 \mathrm{~m} / \mathrm{s}$ at $t / T=0.5-1.0$, respectively. The time-mean velocity magnitudes in the given point are plotted in Fig. 5 too: they are $0.0042 \mathrm{~m} / \mathrm{s}$ and $0.0045 \mathrm{~m} / \mathrm{s}$ for $\mathrm{CH}$ and $\mathrm{PH}$, respectively - i.e. the time-mean velocity generated by $\mathrm{PH}$ is by $8 \%$ higher in the given point.

The time behaviors from Fig. 5 were analyzed to obtain the power spectral density (PSD) and Fig. 6 shows the results. For $\mathrm{CH}$ case, the spectrum low frequency part indicates that the natural frequency is approximately $0.5 \mathrm{~Hz}$. Based on this finding, the thermal actuation was chosen as a subharmonic action with the driven frequency of $0.25 \mathrm{~Hz}$. Evidently, the peak in the low frequency part of the spectrum for PH well confirms this value.

Figure 7 shows the cross-stream velocity profiles at $x=10 \mathrm{~mm}$, i.e. $8.3 \mathrm{D}$. A comparison of the time-mean velocity magnitude of $\mathrm{CH}$ and $\mathrm{PH}$ show that the $\mathrm{CH}$ case

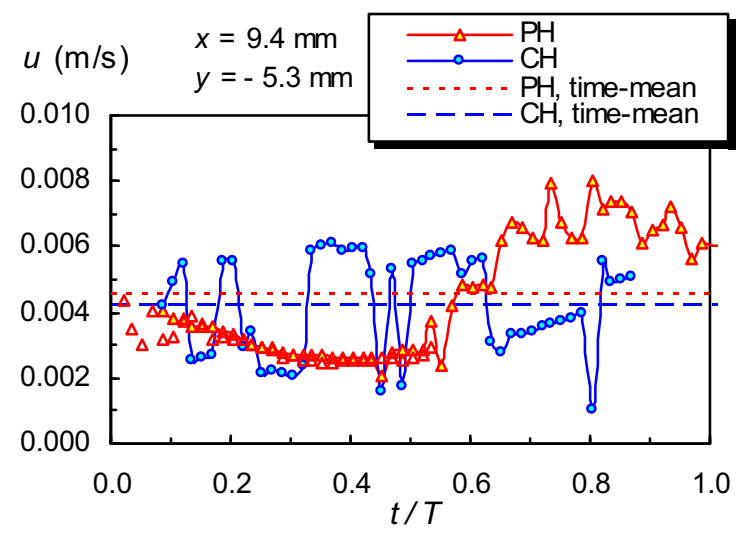

Fig. 5. Temporal evolutions of the velocity magnitude at the point marked by small white rings in Figs. 3 and $4(x=$ $9.4 \mathrm{~mm}, y=-5.3 \mathrm{~mm})$. exhibits by $15 \%$ higher velocity peaks. To better illustrate PH velocity cycle, the velocity magnitude profiles for heating and non-heating parts of the pulsating-supply cycle were evaluated from PIV data presented in Figs. 4(b and c). Figure 7(a) shows that

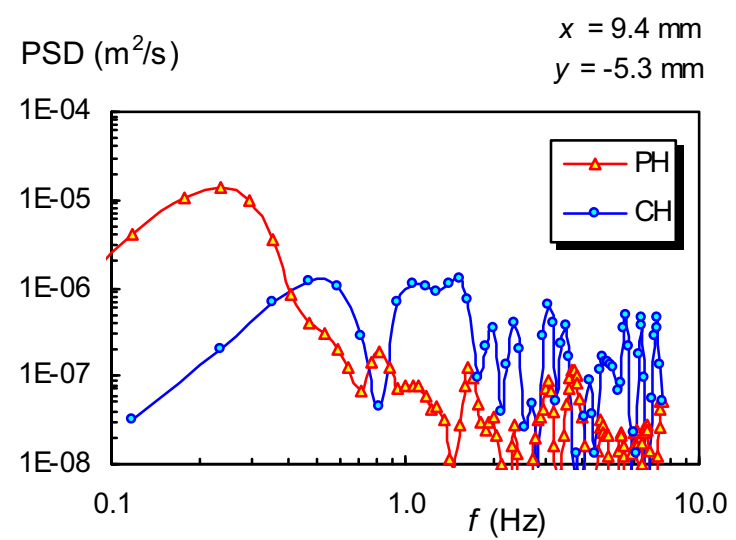

Fig. 6. PSD evaluated from the velocity temporal evolutions presented in Fig. 5.

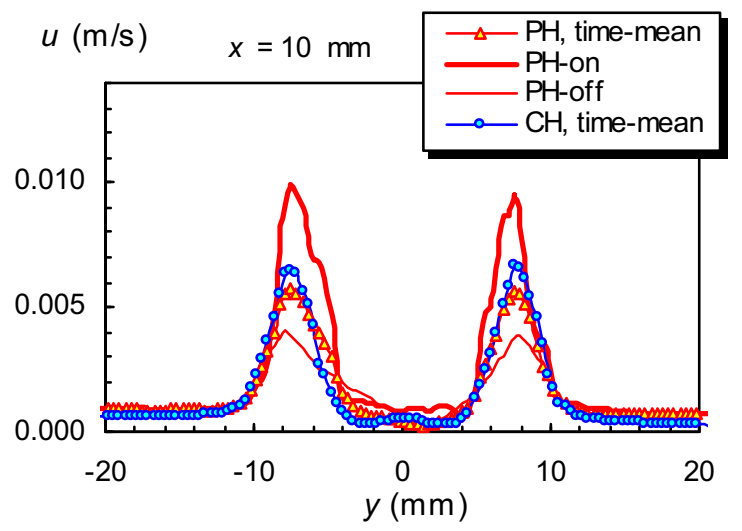

Fig. 7. Cross-stream velocity profiles at $x=10 \mathrm{~mm}$.

velocity magnitude of $\mathrm{PH}$ significantly oscillates along the time-mean value $(0.0057 \mathrm{~m} / \mathrm{s})$ - namely, the peak velocities oscillate from $-30 \%$ to $+70 \%$.

Figure 8 shows another presentation of PIV data from Figs. 3 and 4: the streamwise velocity profiles are plotted along the lines, where the peaks of the time-mean velocity are achieved. Namely the velocity magnitude

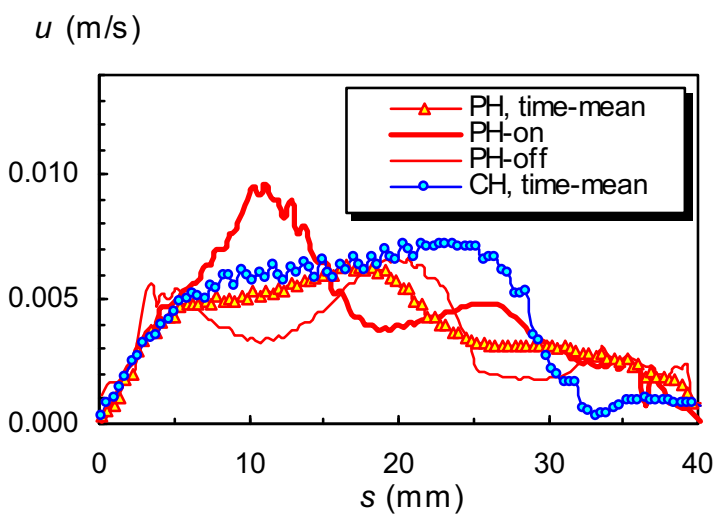

Fig. 8. Streamwise velocity profiles plotted along the line of the time-mean velocity magnitude peaks. 


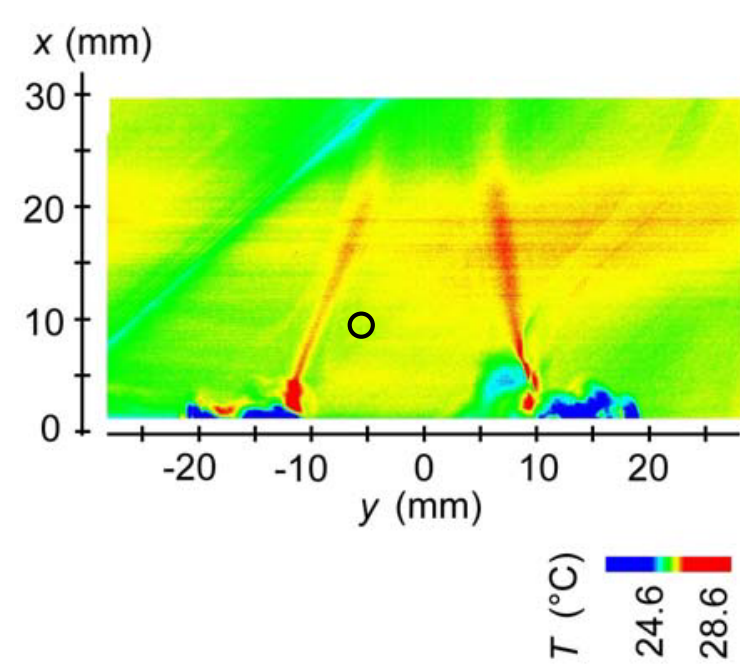

Fig. 9. Time-mean temperature distribution of $\mathrm{CH}$ case; parameters - see Table 1 .

maxima of the left plumes from Figs. 3 and 4 were extracted along the line, which originated at the cylinder vicinity and which is inclined by approximately $20^{\circ}$ from the vertical direction to the right. The length coordinate along this line is named $s$. Figure 8 shows that the velocity of the $\mathrm{CH}$ case gradually increase to the maximum of approximately $0.0072 \mathrm{~m} / \mathrm{s}$ at $s=20-25$ $\mathrm{mm}$. Then a rapid velocity decrease occurs. This decrease is rather a manifestation of the plume turning to the water surface, than a plume flow decay during its streamwise development (it is obvious from Figs. 3 and 4).

Similarly, the PH plume velocity gradually increases to the maximum at $s=17-20 \mathrm{~mm}$, then a rapid velocity decrease occurs as a manifestation of the plume turning. The largest oscillations in Fig. 8 are indicated at $s=11 \mathrm{~mm}$, where the peak velocities oscillate from $-37 \%$ to $+78 \%$. It reasonably agrees with Fig. 7 : very strong velocity oscillations of $\mathrm{PH}$ case occur in the near field at approximately $x=8.3 \mathrm{D}$.

\subsection{Temperature field: PLIF experiment}

Figure 9 shows a typical PLIF result of the twin plumes generated by continuous heating $(\mathrm{CH})$ as the time-mean temperature distribution. Figure 9 was made by averaging over 50 instantaneous temperature field records.

Unlike the velocity flow filed shown in Fig. 3, the temperature field is equalized much faster. Despite the original temperature difference is rather big $\left(T_{\mathrm{w}}-T_{\infty}=\right.$ $19.8{ }^{\circ} \mathrm{C}$, see Table 1), the maximum differences between the plume and its surroundings ale less than $3-4{ }^{\circ} \mathrm{C}$. Moreover, the temperature differences diminish during a distance of $x=0-25 \mathrm{~mm}$, hence a thermal trace of the plume is not identifiable for larger distances $x>25 \mathrm{~mm}$. Therefore Figs. 9 and 10 show a detail of the test section up to $x=0-30 \mathrm{~mm}$ only.

A comparison of the both time-mean cases $\mathrm{CH}$ and PH (Figs. 9 and 10(a), respectively) indicate a basically different character of the both thermal fields. While the
$\mathrm{CH}$ case manifests a monotonically decreasing temperatures in both streamwise and cross-stream directions, the $\mathrm{PH}$ case shows warmed fluid volumes in ascending motion - see the mushroom-like shaped volumes in Fig. 10(a). To indicate this fact more clearly, Fig. 10(b) shows an instantaneous temperature field at the heating moment of the cycle (approximately at $t / T \sim$ 0.8 ). The result is consistent with the above discussed findings of the PIV experiments - cf. Figs. 3 and 4(b).
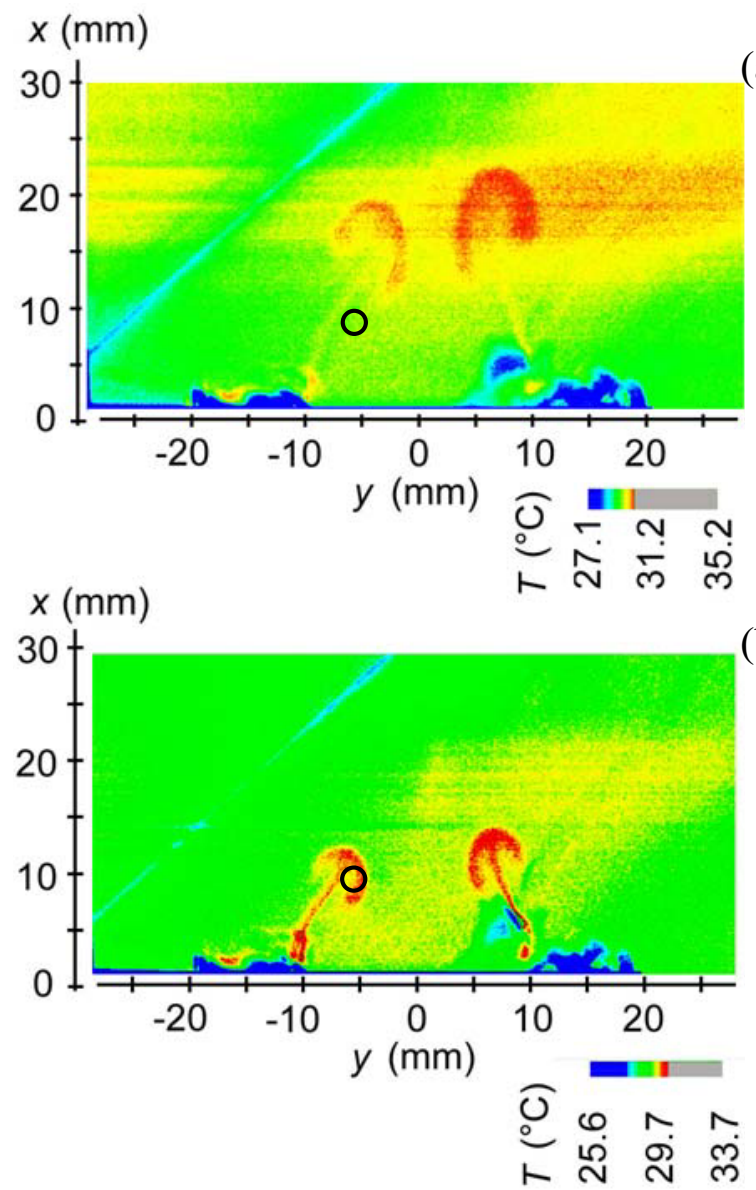

(b)

Fig. 10. Temperature distribution of $\mathrm{PH}$ case; (a) time-mean results, (b) instantaneous temperature field at the heating part of the cycle.

The $\mathrm{PH}$ case concentrates the heating input power into one half of the cycle. It implies an enhancement of heat and mass transfer processes. Therefore higher velocities and higher local temperature gradients can be achieved at $\mathrm{PH}$, in comparison with $\mathrm{CH}$.

Note that the oblique lines in Figs. 9 and 10 are just a consequence of an optical disturbances (mainly reflections from the water surface at $x \sim 40 \mathrm{~mm}$ ), which penetrate across the whole test section as unwanted defects. Certainly, they are not linked with the investigated task.

In Figs. 9 and 10, a small black ring indicates the position of $x=9.4 \mathrm{~mm}, y=-5.3 \mathrm{~mm}$ in which the temperature data will be analyzed in the following text (the same point was used for the PIV data analysis, as was discussed in the text above - see Figs. 3 and 4). A time behavior of the time-mean temperature in this point is shown in Fig. 11. The temperature of the $\mathrm{PH}$ case is a 
bit higher than that of the $\mathrm{CH}\left(30.9{ }^{\circ} \mathrm{C}\right.$ and $27.3^{\circ} \mathrm{C}$, respectively). A reason is a bit higher bulk temperature in the water tank during the $\mathrm{PH}$ experiment. Obviously, the both temperatures oscillate along their time-mean value. Surprisingly, a low frequency thermal actuation cannot be found in Fig. 11 - contrary to the well identifiable period of $\mathrm{PH}$ case in the velocity time record in Fig. 5.

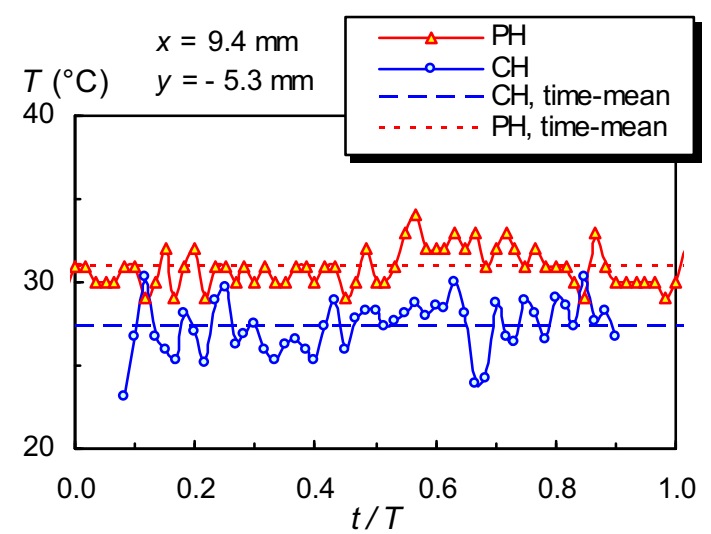

Figure 11. Temporal evolutions of temperatures at the point marked in Figs. 9 and $10(x=9.4 \mathrm{~mm}, y=-5.3 \mathrm{~mm})$.

Figures 12 ( $\mathrm{a}$ and $\mathrm{b}$ ) show the spatial distribution of the time-mean temperature of the both cases $\mathrm{CH}$ and $\mathrm{PH}$, similarly as above discussed distributions of velocities in Figs. 7 and 8. Similarly as in Fig. 11, a bit higher time-mean temperature of $\mathrm{PH}$ in Fig. 12(a) is a
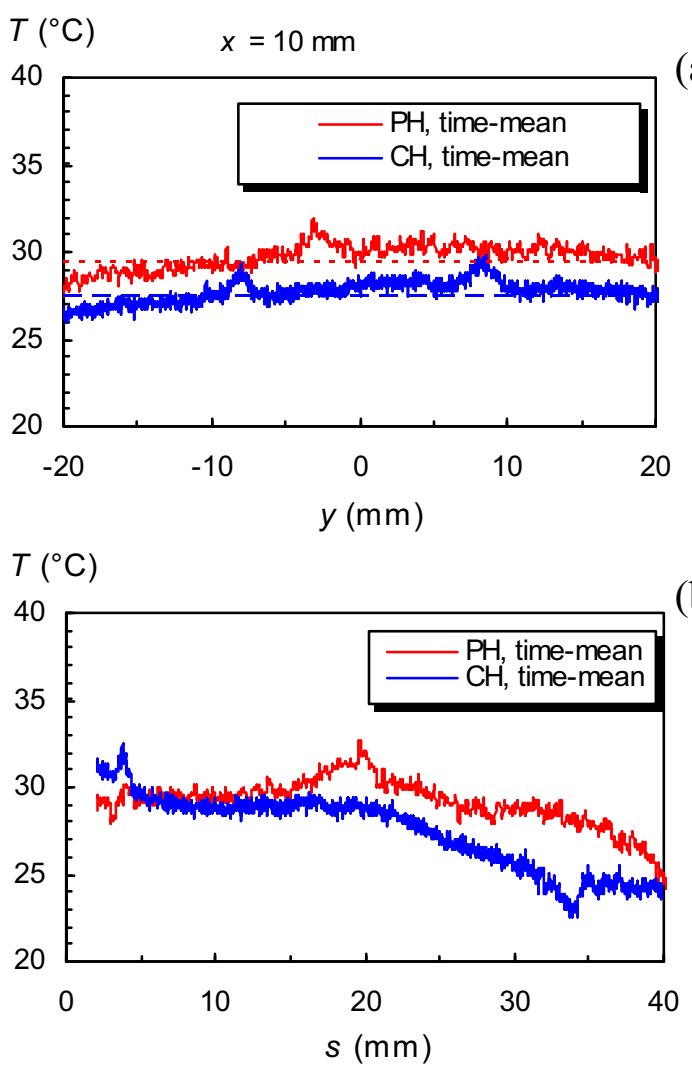

Fig. 12. Temperature profiles, (a) cross-stream profiles at $x=$ $10 \mathrm{~mm}$. (b) streamwise profiles plotted along the line of the temperature peaks. consequence of a bit higher bulk temperature in the water tank during the $\mathrm{PH}$ experiment. More important are the local maxima on the temperature profiles in Fig. 12(a), because they are linked with the investigated task: For the $\mathrm{CH}$ case, two local maxima occur at $y=-8.1 \mathrm{~mm}$ and $+8.2 \mathrm{~mm}$. These maxima are clearly distinguishable a local preheating is about $1.5^{\circ} \mathrm{C}$ for both of them. They indicate crossings of the plumes through the line of $\mathrm{x}=$ $10 \mathrm{~mm}$ - consistently with Fig. 9.

Note that no similar pair of the local maxima exists for the PH case in Fig. 12(a), because of different character of warmed fluid propagation. Namely, an unsteady ascending motion of mushroom-like shaped warmed volumes was shown in Figs. 10( $a$ and b).

Figure 12 (b) shows the streamwise time-mean temperature profiles plotted along the lines, where the peaks of the time-mean temperature are achieved (similarly, the streamwise velocity profiles were shown in Fig. 8). For a small distance $x<15 \mathrm{~mm}$, the timemean temperature of the $\mathrm{CH}$ a $\mathrm{PH}$ cases are nearly the same. For $x>15 \mathrm{~mm}$, the $\mathrm{CH}$ temperatures go to decline. A similar decline of $\mathrm{PH}$ temperatures occurs $10 \mathrm{~mm}$ later. This behavior is another manifestation of an advection unsteady mechanism of ascending motion of warmed fluid volumes. The local maximum on the $\mathrm{PH}$ temperature curve at $x \sim 20 \mathrm{~mm}$ well indicates this warmed fluid volume - consistently with the mushroomlike shaped volume in the left plume in Fig. 10(a).

\section{Conclusions}

A pair of line thermal sources (electrically heated cylinders with a diameter of $D=1.21 \mathrm{~mm}$ ) were used to generate a twine of the plane plumes in water. The distance between the cylinders was $17.9 \mathrm{D}$. Either continuous or pulsating heating were used with the same heating input power $17.4 \mathrm{~W}$. Velocity and temperature fields were investigated by means of PIV and PLIF experiments, respectively.

For the investigated geometry with a moderately small cylinder-to-cylinder distance, both plumes influence each other. Namely, deflections of plumes from a vertical direction occur and the plumes are inclined together. This behavior is caused by effects of fluid entrainments from surroundings versus a confined entrainment from a space between the both plumes.

For a continuous heating, low frequency oscillations were identified and the natural frequency of the plume was evaluated as $0.5 \mathrm{~Hz}$. To promote the unsteady flow character, pulsating heating was used at the subharmonic frequency of $0.25 \mathrm{~Hz}$.

While the maximum time-mean velocity magnitude at the continuous and pulsating heating were commensurable (approximately $0.007 \mathrm{~m} / \mathrm{s}$ ), the pulsating heating causes significant oscillations of the flow field. Namely, the velocity peaks at pulsating heating were by $36 \%$ higher than the velocity peaks at continuous heating. A very strong velocity oscillations were generated by the pulsating heating in the near field at the distance approximately $8.3 \mathrm{D}$ above the cylinders, 
where the velocity maxima oscillate along the time-mean value $0.0057 \mathrm{~m} / \mathrm{s}$ from $-30 \%$ to $+70 \%$.

Behavior of temperature fields consistently agrees with this findings, despite a relatively fast equalization of the temperature field was concluded. The results demonstrate possibilities of a pulsating control on the flow and thermal fields. The pulsations implied an enhancement of heat and mass transfer processes, therefore higher velocities and higher local temperature gradients were achieved

\section{Acknowledgments}

The authors gratefully acknowledge the support from the Grant Agency CR-the Czech Science Foundation (Projects No. 14-08888S and 16-16596S) and the institutional support RVO:61388998.

\section{References}

1. M. H. Schlichting, K. Gersten, Boundary-Layer Theory (8th ed., Springer-Verlag, Berlin, 2000, 1st ed in German: H. Schlichting, Grenzschicht-theorie, Braun, 1951).

2. R. D. Blevins, Applied Fluid Dynamics Handbook (Florida: Krieger Publishing Company 2003)

3. Z. Broučková, S-S. Hsu, A.-B.Wang, and Z.Trávníček, EPJ Web of Conferences 11402007 (2016)

4. H.J. Seuntiëns, R.N. Kieft, C.C.M. Rindt, and A.A. van Steenhoven, Exp. Fluids 31 pp. 588-595 (2001)

5. Z. Broučková and Z. Trávníček, Proceedings of the 12th International Conference on Heat Transfer, Fluid Mechanics and Thermodynamics HEFAT2016 (Malaga, Spain, July 11-13, 2016, pp. 1791-1796)

6. A. P. Vouros, T. Panidis, A. Pollard, R. Schwab, Int. J. Heat Fluid Flow 51 pp. 383-394 (2015) 